\title{
Growth Plate and Cellular Growth
}

\author{
Andrei Chagin ${ }^{a}$, Jovanna Dahlgren ${ }^{b}$ and Lars Sävendahla \\ apediatric Endocrinology Unit, Department of Woman and Child Health, Karolinska Institutet, \\ Stockholm, Sweden \\ ${ }^{b}$ Göteborg Pediatric Growth Research Center, Institute for the Health of Women and Children, \\ The Sahlgrenska Academy at Göteborg University, Göteborg, Sweden
}

\begin{abstract}
The growth plate is a highly organized cartilage structure with a complex cellular biology. Studies have been limited by the relative lack of relevant experimental models. Thanks to new technologies, e.g. targeted deletions of genes, the knowledge is rapidly expanding. The first paper representing the mechanism of the year, describes a novel role of cGMP-dependent protein kinase type II (cGKII) as a molecular switch between proliferative and hypertrophic chondrocytes. Under new paradigms, we selected a paper showing that the fate of chondrocytes to differentiate either into articular or hypertrophic chondrocytes is dependent of the chondrocyte-extracellular matrix interaction and two other papers revealing a role for matrix metalloproteinase-13 (MMP13) in the regulation of longitudinal growth. The new hope section is represented by an experimental study showing that the treatment with IGF-I or anti-cytokines prevents growth retardation induced by pro-inflammatory cytokines normally up-regulated in chronic inflammatory diseases. A new concern is the report of Venn et al. suggesting that high-dose estrogen treatment given to induce premature growth plate fusion in adolescent girls may reduce fertility later in life. Concepts revised or re-centered are represented by two papers describing a mutant human IGF-I associated with growth and developmental abnormalities and an article showing that IGF-I modulates its activity in growth plate chondrocytes by the synthesis of both inhibitory (IGFBP-3) and stimulatory (IGFBP-5) binding proteins. Important observation for clinical practice is represented by a report suggesting improved final adult height in children with congenital adrenal hyperplasia treated with GH and LHRH analogue. Under clinical trials, we selected a report of cartilage oligometric matrix protein (COMP) as a GH-responsive protein in short children treated with GH. New mechanisms are represented by three papers demonstrating: (I) that the $\mathrm{G}$ protein $\alpha$-subunit (Gs $\alpha$ ) inhibits chondrocyte differentiation and is mediating part of the PTH/PTHrP receptor signaling in growth plate chondrocytes; (II) that histone deacetylase 4 controls chondrocyte hypertrophy during skeletogenesis, and (III) that Bmpr1a and Bmpr1b have overlapping functions and are essential for chondrogenesis in vivo. Under new genes, we chose an article reporting that $\mathrm{Wnt} / \beta$-catenin signaling regulates chondrocyte phenotype, maturation, and function in a developmentally regulated manner. The section new hormone includes the demonstration of the SHOX protein in the human growth plate. The review of the year entitled 'Estrogen regulation of growth hormone action' details recent knowledge demonstrating that estrogen affects $\mathrm{GH}$ action also at the level of receptor expression and signaling. Finally, we selected a paper supplying some food for thought, which for the first time demonstrates the expression of Ghrelin by growth plate chondrocytes and suggests novel potential roles for this hormone in cartilage homeostasis.
\end{abstract}

\section{Mechanism of the year}

\section{Cyclic GMP-dependent protein kinase II is a molecular switch from proliferation to hypertrophic differentiation of chondrocytes}

Chikuda H, Kugimiya F, Hoshi K, Ikeda T, Ogasawara T, Shimoaka T, Kawano H, Kamekura S, Tsuchida A, Yokoi N, Nakamura K, Komeda K, Chung U-I, Kawaguchi H

Department of Sensory and Motor System Medicine, Faculty of Medicine, University of Tokyo, Bunkyo, Tokyo, Japan

kawaguchi-ort@h.u-tokyo.ac.jp

Genes Dev 2004;18:2418-2429

Background: The transition from proliferative to hypertrophic chondrocytes is a crucial step in the regulation of longitudinal growth. However, the mechanisms regulating this process are mainly unknown. 
Methods: The Komeda miniature rat Ishikawa (KMI) is a naturally occurring mutant caused by an autosomal recessive mutation, mri, which exhibits longitudinal growth retardation. Analyses of KMIs growth plate morphology as well as protein expression were performed. Growth plate chondrocytes were isolated and transfection experiments were performed ex vivo.

Results: The mri mutation was identified as a deletion in the rat gene encoding cGMP-dependent protein kinase type II ( $c G K I I)$. KMIs showed an expanded growth plate and impaired bone healing with abnormal accumulation of post-mitotic but non-hypertrophic chondrocytes. Ex vivo culture of KMI chondrocytes reproduced the differentiation impairment, which was restored by introducing the adenovirus-mediated $c$ GKII gene. The expression of Sox9, an inhibitory regulator of hypertrophic differentiation, persisted in the nuclei of post-mitotic chondrocytes of the KMI growth plate. Transfection experiments in culture systems revealed that cGKII attenuated the Sox9 functions to inhibit the hypertrophic differentiation of chondrocytes. This attenuation of Sox9 was due to the cGKII inhibition of nuclear entry of Sox9. The impaired differentiation of cultured KMI chondrocytes was restored by the silencing of Sox9 through RNA interference.

Conclusion: The present study for the first time describes a novel role of cGKII as a molecular switch, between proliferative and hypertrophic chondrocytes, through attenuation of Sox 9 function.

During the last few years, our understanding of the molecular mechanisms regulating longitudinal bone growth has greatly increased. The identification of a new gene, cGMP-dependent protein kinase type II (cGKII), to be responsible for the molecular switch between proliferation and differentiation of growth plate chondrocytes has greatly improved our understanding of the regulation of the growth plate. The described mechanism of cGKII action is very interesting; cGKII inhibits nuclear entry of Sox 9 transcription factor and thus promotes chondrocyte differentiation. It is known that parathyroid hormone-related peptide (PTHrP) prevents differentiation of proliferative chondrocytes to become hypertrophic by activation of Sox9. Thus, cGKII and PTHrP act as accelerator/decelerator of chondrocyte differentiation through Sox9. However, one cannot exclude that the growth retardation observed in the KMI rats, at least partially, is due to lower GH production as previously described [1]. Moreover, the differences in growth plate phenotype observed between KMIs and CGKII knockout mice [2] need to be further investigated. The development of a growth plate-specific CGKII knockout mouse model and of course the first description of a human phenotype caring a mutated cGKII mutation would define the exact role of CGKII in the regulation of longitudinal growth.

\section{New paradigms}

\section{Coordination of chondrocyte differentiation and joint formation by $\alpha 5 \beta 1$ integrin in the developing appendicular skeleton}

Garciadiego-Cazares D, Rosales C, Katoh M, Chimal-Monroy J

Departamento de Biología Celular y Fisiología, Instituto de Investigaciones Biomédicas,

Universidad Nacional Autónoma de México, Mexico City, Mexico

jchimal@servidor.unam.mx

Development 2004;131:4735-4742

Background: The events triggering the cartilage differentiation program or the joint formation program are unknown.

Methods: Microinjections of $\alpha 5 \beta 1$ integrin-specific antibodies or RGD peptide (arginine-glycine-aspartic acid) were performed into complete embryos of embryonic day 14.5. Growth plate morphology and levels of chondrocyte proliferation and apoptosis were analyzed. Gene expression within the growth plate was analyzed by in situ hybridization.

Results: Blocking of $\alpha 5 \beta 1$ integrin induced inhibition of prehypertrophic chondrocyte differentiation and ectopic joint formation between proliferating chondrocytes and hypertrophic chondrocytes. Several joint markers, such as Wnt14, Gdf5, chordin, autotaxin, type I collagen and CD44 were found to be expressed in the new ectopic joint. Expression of Indian hedgehog and type II collagen was down-regulated in the newly formed joint. The presence of BMP7 did not affect the formation of the ectopic joint 
between proliferating and hypertrophic chondrocytes if $\alpha 5 \beta 1$ integrin was inhibited. In contrast, misexpression of $\alpha 5 \beta 1$ integrin resulted in joint fusion and formation of prehypertrophic chondrocytes. Conclusion: The decision to form pre-joint or prehypertrophic cartilage is made on the basis of the presence or absence of $\alpha 5 \beta 1$ integrin on chondrocytes.

Longitudinal bone growth is dependent on the level of chondrocyte proliferation, differentiation and matrix production. Differentiation of chondrocytes and matrix production within the growth plate are two interrelated processes. Interactions between the extracellular matrix (ECM) and chondrocytes are well known. However, this study for the first time demonstrates that the fate of chondrocytes to differentiate either into articular or hypertrophic chondrocytes is dependent of the chondrocyte-ECM interaction. This represents a new paradigm for how chondrocyte differentiation is regulated. While integrin $\beta 1$ is expressed throughout the body, $\alpha$-integrins are tissue-specifically expressed. Mutation in the muscle-specific $\alpha 7$ integrin gene results in congenital myopathy and muscle dystrophy in both mice and humans [3]. It could therefore be speculated that mutations in the $\alpha 5 \beta 1$ integrin gene would lead to skeletal abnormalities. However, this needs to be proven.

\section{Critical roles for collagenase-3 (MMP13) in development of growth plate cartilage and in endochondral ossification}

Inada M, Wang Y, Byrne MH, Rahman MU, Miyaura C, Lopez-Otin C, Krane SM

Center for Immunology and Inflammatory Diseases, Department of Medicine,

Harvard Medical School and Massachusetts General Hospital, Boston, Mass., USA

krane.stephen@mgh.harvard.edu

Proc Natl Acad Sci USA 2004;101:17192-17197

Background: Matrix metalloproteinase-13 (MMP13 or collagenase-3) is a member of the neutral endopeptidase family. It is expressed in the skeleton during embryonic development, in human carcinomas, chondrocytes and synovial cells in rheumatoid arthritis and osteoarthritis.

Methods: Growth plate morphology, protein level and RNA expression were analyzed in MMP13 null mice.

Results: MMP13 null mice showed markedly increased length of their hypertrophic zone. Delay in endochondral ossification and formation and vascularization of primary ossification centers was also observed in MMP13 null mice. Absence of MMP13 resulted in significant interstitial collagen accumulation due, in part, to the lack of appropriate collagenase-mediated cleavage.

Conclusion: The paper demonstrates a role of MMP13 in skeletal development and shows involvement of MMP13 in proper cartilage matrix degradation.

\section{Altered endochondral bone development in matrix metalloproteinase-13-deficient mice}

Stickens D, Behonick DJ, Ortega N, Heyer B, Hartenstein B, Yu Y, Fosang AJ, Schorpp-Kistner M, Angel P, Werb Z

Department of Anatomy and Biomedical Sciences Graduate Program, University of California, San Francisco,

Calif., USA

domi@itsa.ucsf.edu; zena@itsa.ucsf.edu

Development 2004;131:5883-5895

Background: The assembly and degradation of extracellular matrix (ECM) molecules are crucial processes during bone development. There are several proteinases expressed during endochondral ossification, including matrix metalloproteinases (MMP)-8, -13 and -14. However, the role of MMP13 has not yet been elucidated.

Methods: MMP13, MMP9 and double MMP13/MMP9 knockout mice were used in this study. Additionally, chondrocyte-specific (collagen II) and osteoblast-specific (collagen I) MMP13 knockouts were analyzed. Limb development, growth plate morphology and other parameters of longitudinal growth were analyzed in all knockout mice.

Results: Inactivation of MMP13 in mice led to abnormal growth plate development. Chondrocyte proliferation and differentiation was unaffected in MMP13 null mice, but their exit from the growth plate 
was delayed. The severity of the MMP13 null growth plate phenotype increased until about 5 weeks and completely resolved by 12 weeks of age. MMP13 null mice had increased trabecular bone, which persisted for months. Tissue-specific knockout of MMP13 in chondrocytes and osteoblasts showed that an increase in trabecular bone occurs independently of the improper cartilage ECM degradation caused by MMP13 deficiency in the growth plate. It was identified that in vivo substrates for MMP13 are collagen type II and aggrecan. Mice lacking both MMP13 and MMP9 had drastically shortened bones, characterized by diminished ECM remodeling, prolonged chondrocyte survival, delayed vascular recruitment and defective trabecular bone formation.

Conclusion: These data support the hypothesis that proper ECM remodeling is a crucial process for programmed cell death, angiogenesis and osteoblast recruitment during normal skeletal development. Moreover, degradation of cartilage collagen and aggrecan is a coordinated process in which MMP13 works synergistically with MMP9.

The extracellular matrix (ECM) is an important component of the growth plate, being unique for each of its layers, and essential for it function. The ECM contains several active metalloproteinases, which take part in ECM degradation. These two articles delineate the role for matrix metalloproteinase-13 (MMP13) in the regulation of longitudinal growth. MMP13 mainly degrades collagen 2, and the knockout of MMP13 resulted in an extended hypertrophic zone early in life which was due to impaired cartilage resorption. These abnormalities were diminishing over time, suggesting some counteracting mechanisms. Bone length was either not affected (Stickens et al., abstract \#2) or only mildly decreased ( $8 \%$; Inada et al., abstract \#1) in the MMP13 null mice. One could speculate that by time accumulation of proteinases secreted by osteoclasts and hematopoietic cells, such as MMP14 and MMP8, would counteract the effects of the missing MMP13. Only double MMP9 plus MMP13 null mice showed remarkable bone shortening. These observations suggest that matrix degradation is a relatively safe process, which involves several interchangeable proteases with overlapping functions. From another angle, the data clearly show that proper degradation of cartilage ECM is crucial for cartilage resorption, angiogenesis and new bone formation. Interestingly, a form of human chondrodysplasia, the Missouri variant of spondyloepimetaphyseal dysplasia, is caused by a mutation in MMP13 [4]. As is the case in MMP13 null mice, the skeletal abnormalities of the Missouri spondyloepimetaphyseal dysplasia change over time. From these two papers, we can learn that proper endochondral ossification requires not only assembly of cartilage matrix but also its normal degradation.

\section{New hope}

\section{Interleukin-1 $\beta$ and TNF- $\alpha$ act in synergy to inhibit longitudinal growth in fetal rat metatarsal bones}

Mårtensson K, Chrysis D, Sävendahl L

Pediatric Endocrinology Unit, Department of Woman and Child Health, Karolinska Institutet, Stockholm, Sweden

lars.savendahl@kbh.ki.se

J Bone Miner Res 2004;19:1805-1812

Background: Impaired growth is common in children with chronic inflammatory diseases such as Crohn's disease or juvenile rheumatoid arthritis. The inflammatory process itself, which includes up-regulation of the pro-inflammatory cytokines interleukin (IL)-1 $\beta$, IL-6, and TNF- $\alpha$, is believed to be at least partly responsible for the poor growth in these patients. The authors aimed to clarify whether these cytokines can act locally in the growth plate to suppress longitudinal growth and whether any negative effects can be reversed.

Methods: The effects of cytokines on longitudinal bone growth were studied in fetal (day E20) rat metatarsal bones kept in culture. After being cultured for 7 days, the bones were sectioned, and chondrocyte proliferation was assessed by bromodeoxyuridine (BrdU) incorporation and apoptosis by TUNEL assay.

Results: When added separately, IL-1 $\beta$ and TNF- $\alpha$ impaired longitudinal bone growth only at a high concentration $(100 \mathrm{ng} / \mathrm{ml}$ each). In contrast, when added in combination, IL-1 $\beta$ and TNF- $\alpha$ potently 
inhibited growth at far lower concentrations (from $3 \mathrm{ng} / \mathrm{ml} \mathrm{each}$ ) and also decreased chondrocyte proliferation and increased apoptosis. Growth failure induced by the combination of IL-1 $\beta$ and TNF- $\alpha$ could be counteracted by anti-IL-1 $\beta$, anti-TNF- $\alpha$, or IGF-I. IL- 6 did not affect longitudinal growth even when added in combination with IL-1 $1 \beta$ or TNF- $\alpha$.

Conclusion: IL-1 $\beta$ and TNF- $\alpha$ act in synergy to locally suppress longitudinal growth, an effect that can be partially reversed by IGF-I. Although growth hormone (GH)/IGF-I may improve longitudinal growth in children with chronic inflammatory diseases, our results suggest that the inflammatory process itself must be targeted to achieve normal growth.

Chronic inflammation may lead to malnutrition, which can contribute to the impaired growth often seen in these patients. Nevertheless, many children fail to improve their growth and achieve their growth potential despite nutritional intervention, suggesting that the inflammation, by itself, inhibits longitudinal growth. Cytokines are normally up-regulated during chronic inflammation, and may affect growth either through a systemic effect and/or through a local effect on the growth plate. Using a model of cultured fetal rat metatarsal bones, the authors demonstrate that IL-1 $\beta$ and TNF- $\alpha$ act in synergy to induce growth arrest by directly targeting the growth plate cartilage, an effect which was linked to a decrease in chondrocyte proliferation and induced apoptosis. These effects could be partly reversed by treating the bones with specific antibodies to IL- $1 \beta$ or TNF- $\alpha$. It can therefore be hypothesized that anti-cytokine treatment could improve growth in children with chronic inflammatory diseases. Data on that effect is limited, and the efficacy of this treatment on growth and final height has to be established $[5,6]$. The authors could only partly reverse cytokine-induced growth retardation by IGF-I, emphasizing the importance to target the inflammatory process itself to optimize growth in children with chronic inflammatory diseases. Based on the data presented in this paper, it can be speculated that the combination of IGF-I and anti-cytokine treatment could further improve growth in patients with chronic inflammatory diseases. However, it should be emphasized that this is an animal experiment, and human studies are needed to verify such an approach.

\section{New concerns}

\section{Estrogen treatment to reduce the adult height of tall girls: long-term effects on fertility}

Venn A, Bruinsma F, Werther PG, Pyett P, Baird D, Jones P, Rayner J, Lumley PJ

Menzies Research Institute, University of Tasmania, Hobart, Australia

Alison.Venn@utas.edu.au

Lancet 2004;364:1513

Background: Treatment with high-dose estrogen to reduce adult height of tall girls has been practiced since the 1950 s.

Methods: This is a retrospective cohort study to assess the long-term effects of this treatment on fertility. Participants were identified from the records of Australian pediatric endocrinologists who assessed tall girls from 1959 to 1993, and from self-referrals. The study included girls who had received estrogen treatment (diethylstilbestrol or ethinyl estradiol) and those who were assessed but not treated. Data about reproductive history was collected by telephone interview.

Results: A total of 1432 eligible individuals were identified, of whom 1243 (87\%) could be traced. Of these, $780(63 \%)$ completed the interviews: 651 were identified from endocrinologists' records, 129 were self-referred. There were no differences in socioeconomic and other characteristics between treated $(\mathrm{n}=371)$ and untreated $(\mathrm{n}=409)$ women. After adjustment for age, treated women were more likely to have ever tried for 12 months or more to become pregnant without success (relative risk [RR] 1.80, 95\% CI 1.40-2.30); more likely to have seen a doctor because they were having difficulty becoming pregnant (RR 1.80,1.39-2.32), and more likely to have ever taken fertility drugs (RR 2.05, 1.39-3.04). The treated group was $40 \%$ less likely to conceive in any given menstrual cycle of unprotected intercourse. These associations persisted when self-referred women were excluded. However, at the end, both groups achieved pregnancy at the same rate (66.9 and $65.3 \%$, respectively). 
Conclusion: The data suggest that high-dose estrogen treatment in adolescence reduces female fertility later in life.

It is now almost 40 years since treatment with high-dose estrogen was described to reduce final height in the adolescent tall female [7]. Today, we know that estrogen receptors are expressed in the human growth plate [8], and that a lack of estrogen receptor- $\alpha$ prevents growth plate fusion in humans [9]. Thus, it is undisputable that estrogens play a crucial role in the process of promoting growth plate fusion. Height reductions of $2.1-10.0 \mathrm{~cm}$ have been reported in tall girls treated with high-dose estrogen [10]. It is worth pointing out that the effectiveness of the treatment has not been documented in a single randomized controlled trial. Multiple short-term side effects have been observed including weight gain, nausea, thrombosis and ovarian cysts [10]. Any long-term side effects have been much debated and any strong evidence for such effects are still lacking. This is the first large cohort and long-term follow-up study of reproductive functions in tall girls treated with high-dose estrogen. The authors report that it took longer time to conceive and more fertility services were required in women previously treated with high-dose estrogens. It is unknown whether it was the estrogen exposure during puberty that has affected reproductive potential later in life, or if some of the girls had a common mechanism for tall stature and impaired fertility. However, for women who have had estrogen treatment for tall stature, it is reassuring that the likelihood of eventually conceiving and having a live birth was not significantly lower for the treated than for the untreated women. Further studies focusing on long-term effects of high-dose estrogen treatment are needed. Meanwhile, patients and parents should be informed of these results.

\title{
Concepts revised or re-centered
}

\section{IGF-I stimulates cell proliferation and induces IGFBP-3 and IGFBP-5 gene expression in cultured growth plate chondrocytes via distinct signaling pathways}

\author{
Kiepe D, Ciarmatori S, Hoeflich A, Wolf E, Tonshoff B \\ University Children's Hospital, Heidelberg, Germany \\ Burkhard_Toenshoff@med.uni-heidelberg.de \\ Endocrinology 2005;146:3096-3104
}

Background: In the cellular microenvironment, about $95 \%$ of all IGF-I is bound to IGF-binding proteins (IGFBPs). IGFBPs modulate the bioactivity of IGF-I and can have both stimulatory and inhibitory effects. IGF-I in turn partially regulates the production of IGFBPs. So far, any IGF-mediated regulation of IGFBP production in the growth plate has not been defined.

Methods: Cultures of primary rat epiphyseal chondrocytes and the RCJ3.1C5.18 mesenchymal chondrogenic cell line were used. Different signaling pathways were blocked either by PD098059 or U0126 (p42/44 MAPK pathway inhibitors) or by LY294002 (phosphatidylinositol-3 kinase (PI3K) signaling pathway inhibitor).

Results: IGF-I increased the production of IGFBP-3 and IGFBP-5 by primary rat chondrocytes in a doseand time-dependent manner. Inhibition of the p42/44 MAPK pathway with either PD098059 or U0126 abrogated IGF-I-dependent stimulation of IGFBP-3 mRNA expression but did not affect the increased IGFBP-5 mRNA levels. However, the IGF-I induced increases of both IGFBP-3 and -5 mRNA were blocked when co-incubated with the PI3K inhibitor, LY294002. The IGFBP-5 data were confirmed in the RCJ3.1C5.18 cell line, which does not express IGFBP-3. Finally, the IGF-I-induced IGFBP-5 gene expression was demonstrated to require de novo mRNA transcription and de novo protein synthesis. Conclusion: IGF-I modulates its activity in rat growth plate chondrocytes by the synthesis of both inhibitory (IGFBP-3) and stimulatory (IGFBP-5) binding proteins.

The importance of the GH/IGF-I system for the regulation of longitudinal growth is unequivocal. The majority of GH-dependent growth stimulatory effects are mediated through IGF-I. GH is capable of stimulating IGF-I production not only in the liver but also directly in the growth plate. IGF-I in turn 
regulates its own bioavailability by stimulating the production of IGF-binding proteins. From this study we can learn that IGF-I stimulates chondrocyte production of proliferation-inducing IGFBP-5 and inhibiting IGFBP-3; these effects being mediated through different and only partially overlapping intracellular signaling pathways. The data indicate that IGF-I-induced stimulation of both proand anti-proliferative IGFBPs may lead to a functional equilibrium within the growth plate. However, it should be emphasized that IGF-I not only has proliferative effects, but also promotes chondrocyte differentiation and protects from apoptosis which further complicates the picture. Further studies are needed to define the physiological role of IGFBPs locally produced in the growth plate and how they are regulated by IGF-I. Nevertheless, the finding that IGF-I uses different and only partially overlapping pathways for the regulation of two IGFBPs with opposing biological functions might be important for the modulation of IGF bioactivity in the cellular microenvironment.

\section{Homozygous and heterozygous expression of a novel IGF-I mutation}

Walenkamp MJE, Karperien M, Pereira AM, Hilhorst-Hofstee Y, van Doorn J, Chen JW, Mohan S, Denley A, Forbes B, van Duyvenvoorde HA, van Thiel SW, Sluimers CA, Bax JJ, de Laat JAPM, Breuning MB, Romijn JA, Wit JM

Departments of Pediatrics, Leiden University Medical Center, Leiden, The Netherlands

m.j.e.walenkamp@lumc.nl

J Clin Endocrinol Metab 2005;90:2855-2864

Background: IGF-I is a key factor in intrauterine development and postnatal growth and metabolism. In contrast to childhood and adult life, the secretion of IGF-I during fetal life is not dependent on GH. Previous reports of human IGF-I deficiency or partial IGF-I insensitivity have shown decreased intrauterine growth, which is in contrast to GH-deficient individuals who show only postnatal growth retardation.

Methods: A 55-year-old male, the first child of consanguineous parents, presenting with severe intrauterine and postnatal growth retardation, deafness and mental retardation, was genetically examined. Moreover, investigation of 24 of his family members was also performed.

Results: The index case had elevated GH levels and was found to have a homozygous missense mutation in the IGF-I gene (see below cited paper, Denley et al.). The investigation of relatives revealed 9 of 24 heterozygous members enabling the characterization of the phenotype of IGF-I haploinsufficiency. Their birth weight and head circumference were lower in comparison to non-carriers. Their mean adult height was 0.6 SDS lower than non-carriers, the mean difference in head circumference was 1.5 SDS, and serum IGF-I and fasting insulin levels were increased. No effects were seen on inner ear development. Conclusion: This homozygous missense mutation in the IGF-I gene results in an abnormal IGF-I molecule with low receptor binding causing severe pre- and postnatal growth retardation. Also the haploinsufficiency results in a mild inhibition of intrauterine and postnatal statural and cranial growth.

\section{Structural and functional characteristics of the Val44Met insulin-like growth factor I missense mutation: correlation with effects on growth and development}

Denley A, Wang CC, McNeil KA, Walenkamp MJE, van Duyvenvoorde H, Wit JM, Wallace JC, Norton RS,

Karperien M, Forbes BE

School of Molecular and Biomedical Science, University of Adelaide, Australia

briony.forbes@adelaide.edu.au

Mol Endocrinol 2005;19:711-721

Background: The phenotype resulting from a missense mutation in the IGF-I gene was recently described (see above abstract by Walenkamp et al.).

Methods: A biochemical and structural analysis of Val44Met IGF-I was conducted to provide a molecular basis for the phenotype reported.

Results: The Val44Met IGF-I exhibited a 90-fold decrease in type 1 IGF receptor (IGF-1R) binding compared with wild-type. The ability of Val44Met IGF-I to signal via the extracellular signal-regulated kinase was found to be impaired. Moreover, binding or activation of the insulin receptor was not detectable. This is in contrast to the normal binding capacity to IGF-binding protein-2 (IGFBP-2), 
IGFBP-3, and IGFBP-6 which suggests a maintained overall structure. Structural analysis by nuclear magnetic resonance confirmed retention of near-native structure with only local side-chain disruptions despite the significant loss of function.

Conclusion: This is the first structural study of a naturally occurring mutant human IGF-I associated with growth and developmental abnormalities.

Not only gene deletions, but also missense mutations in the homeodomain may alter the biological effects of hormones and can affect receptor binding and dimerization. This is the first report on a homozygous missense mutation in the human IGF-I gene with a low binding capacity to the IGF-I receptor. The patient, characterized in these two papers, provides an opportunity to evaluate the effects of primary IGF-I insufficiency in adulthood. The phenotype is clearly distinguished from the secondary IGF-I deficiency present in severe GH resistance or deficiency. Yet, the effect of primary or secondary IGF-I insufficiency on postnatal growth seem to be similar. Interestingly, the mutation is relatively well tolerated in adulthood, although the affected individuals develop signs of the metabolic syndrome. The phenotype of this patient has several similarities with the previous reported partial deletion of the IGF-I gene [11]. These two papers expand our understanding of phenotypegenotype features of different mutations in the IGF-I gene.

\section{Important observations for clinical practice}

\section{Treatment with growth hormone and LHRH analogue improves final adult height in children with congenital adrenal hyperplasia}

Lin-Su K, Vogiatzi MG, Marshall I, Harbison MD, Macapagal MC, Betensky B, Tansil S, New MI

Department of Pediatrics, Mount Sinai School of Medicine, New York, N.Y., USA

maria.new@mssm.edu

J Clin Endocrinol Metab 2005;90:3318-3325

Background: Most patients with congenital adrenal hyperplasia $(\mathrm{CAH})$ prematurely complete their growth and ultimately become short as adults. Due to excess androgens, affected children often develop accelerated linear growth during childhood accompanied by premature growth plate fusion.

Methods: Fourteen patients with CAH predicted to be $>1.0$ SD below their midparental target height received GH and LHRH analog (LHRHa) until final height (treatment group). They were matched to a historical group of CAH patients treated with glucocorticoids only (untreated group).

Results: The mean duration of GH treatment was $4.2 \pm 2.0$ years. In the treatment group, the final height SDS $(-0.4 \pm 0.8$ SDS) was significantly greater than both the initial prediction $(-1.5 \pm 0.9$ SDS) and the final height SDS of the untreated group (-1.4 \pm 1.1 SDS). However, there was no statistically significant difference in bone age between the treated and untreated group. Finally, there was a negative correlation between age at start of treatment and gain in height.

Conclusion: The results suggest that the combination of GH and LHRHa may improve final adult height in patients with $\mathrm{CAH}$.

Monitoring glucocorticoid treatment during puberty is critical for the height outcome in individuals with CAH. Two previous reports on successful LHRHa treatment in children with precocious puberty and $\mathrm{CAH}$ have been published $[12,13]$. However, no final height data have been reported yet. The present study is a small, non-randomized study with historical controls followed to final height. The authors have previously published 2-year height data [14]. CAH children treated with the combination of GH and LHRHa improved their final adult height by about 1 SDS or $7 \mathrm{~cm}$ above untreated historical controls. Although the duration of GH + LHRH treatment was short and primarily given during their pubertal years, this is the first study to suggest an improvement in final adult height with the combination of GH and LHRHa in this patient group. It may be speculated that the benefit of GH treatment could be larger if the treatment had been started at an earlier age. The non-randomized design of this study does not permit any concrete recommendations, and new carefully designed studies are needed to confirm these findings. 


\section{Cartilage oligomeric matrix protein increases in serum after the start of growth hormone treatment in prepubertal children}

Bjarnason R, Andersson B, Kim HS, Olsson B, Swolin-Eide D, Wickelgren R, Kristrom B, Carlsson B, Albertsson-Wikland K, Carlsson LM

Göteborg Pediatric Growth Research Center, Department of Pediatrics,

The Institute of the Health of Women and Children, Göteborg, Sweden

ragnar.bjarnason@vgregion.se

J Clin Endocrinol Metab 2004;89:5156-5160

Background: Cartilage oligomeric matrix protein (COMP) is a homopentameric glycoprotein synthesized by chondrocytes and found primarily in the extracellular matrix of cartilage, ligament and tendon. Mutations in the COMP gene have been associated with both pseudoachondroplasia and multiple epiphyseal dysplasia. The molecular mechanisms mediating the effects of GH and IGF-I on bone growth are not fully understood.

Methods: Gene expression screening was performed by microarray analysis in cultures of human primary chondrocytes treated with either GH or IGF-I. The chondrocyte cultures were established from an extirpated extra thumb from a 1-year-old boy. In addition, serum levels of COMP, GH, IGF-I and IGFBP-3 were measured in a group of 113 short prepubertal children treated with GH for at least 1 year. These children had a wide range of endogenous $\mathrm{GH}$ production and included both GH-deficient and non-GH-deficient individuals.

Results: By stimulating primary cultures of human chondrocytes with GH and IGF-I, COMP was identified as a GH- and IGF-I-responsive gene. At baseline, serum COMP concentrations correlated with serum concentrations of IGF-I and IGFBP-3. Mean serum COMP increased during GH treatment and correlated to the individual changes in IGF-I levels.

Conclusion: This paper demonstrates that COMP expression is up-regulated by both GH and IGF-I in primary cultures of human chondrocytes and that serum levels of COMP increase after the initiation of $\mathrm{GH}$ treatment in short children.

Based on results from initial gene expression screening of cultured human chondrocytes, the authors hypothesized that GH and IGF-I effects on bone growth might be mediated through increased production of COMP, a glycoprotein normally expressed by growth plate chondrocytes. To test this hypothesis, serum levels of COMP were measured in short individuals before and during first year of $\mathrm{GH}$ treatment. Study individuals were closely monitored including their height development. Although serum levels of COMP increased during GH treatment, there was no correlation between COMP levels and subsequent growth response in GH-treated short individuals. This study clearly shows a link between GH/IGF-I and COMP production but does not support the measurements of serum COMP as a practical tool to predict growth response to GH treatment. Any clinical use of serum measurements of COMP to evaluate responsiveness to GH treatment need to be documented before applied into clinical practice. 


\section{Chondrocyte-specific knockout of the G protein Gs $\alpha$ leads to epiphyseal and growth plate abnormalities and ectopic chondrocyte formation}

Sakamoto A, Chen M, Kobayashi T, Kronenberg HM, Weinstein LS

Metabolic Diseases Branch, National Institute of Diabetes, Digestive, and Kidney Diseases,

National Institutes of Health, Bethesda, Md., USA

leew@amb.niddk.nih.gov

J Bone Miner Res 2005;20:663-671

Background: Ubiquitously expressed G protein $\alpha$-subunit $(G s \alpha)$ mediates signaling through $\mathrm{G}$ proteincoupled receptors and the cAMP/protein kinase A signaling pathway. The exact role for Gs $\alpha$ in the direct regulation of growth plate development is unclear.

Methods: Bone and cartilage morphology was analyzed in mice with growth plate-specific ablation of $G s \alpha$. In situ hybridization was used to study gene expression.

Results: Mice with chondrocyte-specific inactivation of the Gs $\alpha$ gene died within minutes after birth due to breathing problems. The growth plates of these mice exhibited severe abnormalities including shortening of the proliferative zone and accelerated differentiation of hypertrophic chondrocytes, a phenotype similar to that of PTH/PTH-related peptide (PTHrP) receptor knockout mice. The expression of Indian hedgehog (Ihh) and the PTH/PTHrP receptor in prehypertrophic chondrocytes was unaffected in the chondrocyte-specific Gs $\alpha$ gene knockout mice. PTHrP expression in periarticular cartilage was increased in the mutant mice, probably due to the closer proximity of Ihh-secreting chondrocytes. In addition, these mice developed ectopic cartilage at the anterior side of the metaphyseal region in the tibia. Mice with heterozygote $G s \alpha$ deficiency exhibited no growth abnormalities.

Conclusion: Gs $\alpha$ inhibits chondrocyte differentiation and is a signaling mediator of the PTH/PTHrP receptor in growth plate chondrocytes.

Patients with Albright hereditary osteodystrophy (AHO), who are heterozygous for inactivating Gs $\alpha$ mutations, generally present with an abnormal skeletal phenotype including brachydactyly and short stature. It can therefore be assumed that $G s \alpha$ pathways play important roles in growth plate development. The authors of this paper developed a new mouse line with growth plate-specific inactivation of Gs $\alpha$. These mice, having shortened limbs and domed skull, provide the first direct in vivo evidence that $G s \alpha$ is a negative regulator of chondrocyte differentiation. It should be pointed out that only mice with homozygous mutation of Gs $\alpha$ exhibited growth abnormalities whereas heterozygous mice had normal growth plate development suggesting species differences with regards to Gs $\alpha$ imprinting between humans and mice. Moreover, the data support PTHrP signaling in growth plate chondrocytes mainly to be mediated by Gs $\alpha$. Consequently, it can be speculated that the abnormal skeletal development in AHO patients is a result of disturbed PTHrP signaling secondary to their inactivated Gs $\alpha$. Complete inactivation of PTHrP signaling due to a mutated PTHrP/PTH receptor (Bloomstrand's chondrodysplasia) leads to more severe abnormalities of cartilage development and early death due to respiratory failure. However, as a human homozygous Gs $\alpha$ mutation has not yet been identified, it is still unclear if PTHrP signaling is dependent on Gs $\alpha$ signaling.

\section{Histone deacetylase 4 controls chondrocyte hypertrophy during skeletogenesis}

Vega RB, Matsuda K, Oh J, Barbosa AC, Yang X, Meadows E, McAnally J, Pomajzl C, Shelton JM, Richardson JA, Karsenty G, Olson EN

Department of Molecular Biology, University of Texas Southwestern Medical Center, Dallas, Tex., USA

eric.olson@utsouthwestern.edu

Cell 2004;119:555-556

Background: The tissue-specific histone deacetylases (HDACs) class II (HDAC 4, 5, 7 and 9) regulate gene transcription through histone deacetylation following chromatin condensation. It has previously been 
shown that HDAC9 acts as a negative regulator of cardiomyocyte hypertrophy and skeletal muscle differentiation. Although an extensive in vitro analysis of the consequences of histone acetylation/deacetylation has been performed, little is known about the specific functions of HDACs in vivo.

Methods: HDAC4 null mice and transgenic mice, over-expressing HDAC4 in the growth plate, were used in the study.

Results: HDAC4-null mice displayed premature ossification of developing bones which was due to ectopic and early onset of chondrocyte hypertrophy. In vivo inactivation of HDAC4 caused an increased expression of Runx2 with subsequent over-differentiation of chondrocytes. Growth platespecific over-expression of HDAC4 in vivo completely inhibited chondrocyte differentiation and endochondral ossification. Moreover, transfection of HDAC4 inhibited Runx 2 transcription activity in COS cells. Chromatin immunoprecipitation revealed direct binding between Runx2 and HDAC4.

Conclusion: HDAC4 regulates chondrocyte hypertrophy and endochondral bone formation by inhibiting the activity of Runx2, a transcription factor needed for chondrocyte hypertrophy.

A new player in the regulation of longitudinal bone growth has been identified. The present data suggest that class II HDACs may act within different cell types to control hypertrophic growth in response to developmental or pathological signals. Chondrocyte hypertrophy is essential for vascular invasion, osteoblast differentiation, and endochondral ossification [15]. To date, the only transcription factor shown to be required for chondrocyte hypertrophy is Runt-related transcription factor-2 (Runx2), which is also necessary for osteoblast differentiation [16]. HDACs are well known to modulate cell growth and differentiation by governing chromatin structure and repressing the activity of specific transcription factors. Inactivation experiments of HDAC5 and HDAC9 in mice have previously shown that both HDAC5 and HDAC9 repress cardiac cellular hypertrophy $[17,18]$. In the present study, it was elegantly shown that inactivation of the HDAC4 gene leads to inappropriate chondrocyte hypertrophy and ectopic bone formation. It was also shown that HDAC4 suppresses chondrocyte differentiation and long bone formation through direct inhibition of Runx2. Thus, the knowledge that cellular hypertrophy is regulated by class II HDACs, through their association with specific target transcription factors, could open new strategies to therapeutically manipulate this mechanism. We know of no clinical associations between skeletal abnormalities in humans and HDAC4 mutations. A possible explanation could be that the mutation might be lethal in humans.

\section{Bmpr1a and Bmpr1b have overlapping functions and are essential for chondrogenesis in vivo}

Yoon BS, Ovchinnikov DA, Yoshii I, Mishina Y, Behringer RR, Lyons KM

Departments of Molecular, Cell, and Developmental Biology, University of California, Los Angeles, Calif., USA

klyons@mednet.ucla.edu

Proc Natl Acad Sci USA 2005; 102:5062-5067

Background: Bone morphogenetic proteins (BMPs) promote chondrogenic differentiation in vitro. The constitutively active forms of BMP receptor type 1A (BMPR1A) and 1B (BMPR1B) promote chondrogenesis in transgenic mice. However, any importance of BMP signaling in early chondrogenesis has yet not been defined.

Methods: Mice with growth plate-specific inactivation of Bmpr1a (Bmpr1a $\left.{ }^{C K O}\right)$, Bmpr1b null $\left(B m p r 1 b^{-l-}\right)$ mice and double Bmpr1a ${ }^{C K O} / B m p r 1^{b-l-}$ knockout mice were generated. Bone development was analyzed in all knockout animals.

Results: Bmpr1a ${ }^{C K O}$ or Bmpr1b $b^{-1-}$ knockout mice were able to form intact cartilaginous elements and to develop skeletal elements with mild deformities. However, double Bmpr $1 a^{C K O} / B m p r 1 b^{-1-}$ knockouts developed a severe generalized chondrodysplasia. The majority of bones normally formed through endochondral ossification were absent, and the few ones that were formed were rudimentary. The few cartilage condensations that were formed in the double knockouts were delayed in the prechondrocytic state and an organized growth plate was never formed. The reduced size of cartilage condensations in double knockouts was associated with increased apoptosis and decreased proliferation. The production of extracellular matrix was decreased. No expression of Sox9, L-Sox5, and Sox6 was detectable in precartilaginous condensations in double Bmpr $1 a^{\mathrm{CKO}} / \mathrm{Bmpr} 1 b^{-1-}$ knockout mice. 
Conclusion: BMP signaling is needed for multiple aspects of early chondrogenesis in vivo. Moreover, BMPR1A and BMPR1B are functionally redundant during early chondrogenesis.

Bone morphogenetic proteins (BMPs) are important regulators of longitudinal bone growth, although the specific roles of different BMP receptors are uncertain. The present study clearly show that two such receptors BMPR1A and BMPR1B have overlapping functions during chondrogenesis and that double knockout mice $\left(B m p r 1 b^{-1-}, B m p r 1 a^{-l-}-\mathrm{Co} / 2\right)$ have severe chondrodysplasia with absence of the majority of skeletal elements formed through endochondral ossification. Abolished expression of Sox family transcription factors in the double knockouts suggests that BMP signaling promotes chondrogenesis through up-regulation of the transcription factor Sox9. Of great interest is the fact that all major regulators of chondrogenesis, like TGF $\beta / B M P$ super-family molecules, Wnt family signaling molecules and Indian hedgehog/ /PTHrP), all are converged on the regulation of Sox9 and/or Runx2 transcription factors. Recently described genes that are crucial for bone growth, such as cyclic GMP-dependent protein kinase II (cGKII; see paper by Chikuda $\mathrm{H}$ et al.) and histone deacetylase 4 (HDAC4; see paper by Vega RB et al.), also act through regulation of either Sox9 or Runx2. It is obvious that these two transcription factors are indeed key factors in the regulation of bone development and longitudinal growth.

\section{New genes}

\section{Developmental regulation of Wnt/B-catenin signals is required for growth plate assembly, cartilage integrity, and endochondral ossification}

Tamamura Y, Otani T, Kanatani N, Koyama E, Kitagaki J, Komori T, Yamada Y, Costantini F, Wakisaka S, Pacifici M, Iwamoto M, Enomoto-Iwamoto $\mathrm{M}$

Department of Orthopedic Surgery, Jefferson Medical College, Thomas Jefferson University, Philadelphia, Pa., USA

Motomi.Iwamoto@jefferson.edu

J Biol Chem 2005; 280:19185-19195

Background: It has been shown that Wnt/ $\beta$-catenin signaling is involved in chondrocyte differentiation and bone formation. It acts mainly through two major transcription factors, Sox9 and Runx2. However, depending on different experimental conditions, such as animal models, degree of chondrocyte differentiation and strength of the $\beta$-catenin signaling, Wnt $/ \beta$-catenin could either promote or inhibit chondrocyte differentiation and bone formation. The study was conducted in order to reveal the mechanisms of $\mathrm{Wnt} / \beta$-catenin action in chondrocytes of various degrees of differentiation.

Methods: Transgenic mice were developed in which a constitutively activated form of $\beta$-catenin (CA-LEF) was expressed in nascent chondrocytes in developing mouse embryo skeletal elements using a cartilage characteristic collagen II promoter. Additionally, chick developing limbs were virally transfected with activated $\beta$-catenin and chondrocyte differentiation was analyzed.

Results: Multiple skeletal defects were observed in the transgenic mice. The growth plate organization was totally disturbed including the lack of maturing chondrocytes expressing Indian hedgehog and collagen X. Endochondral ossification was also abrogated. Interestingly, the transgenic cartilaginous elements were ill defined, intermingled with surrounding connective and vascular tissues, and even displayed abnormal joints. However, expression of activated $\beta$-catenin mutant $(\delta$ - $\beta$-catenin) in chondrocytes already engaged in maturation such as those present in chick limbs, further promoted chondrocyte maturation and bone. Differential responses to $\mathrm{Wnt} / \beta$-catenin signaling were confirmed in cultured chondrocytes.

Conclusion: Wnt/ $\beta$-catenin signaling regulates chondrocyte phenotype, maturation, and function in a developmentally regulated manner. This pathway is also involved in the organization of the growth plate and the process of endochondral ossification.

Our understanding of the mechanisms involved in cartilage formation is far from complete. For example, articular and growth plate cartilage are formed from the same cartilage template in every long bone, but have different morphology, organization and functions. The family of $\mathrm{Wnt} / \beta$-catenin 
signaling proteins, which include more than 20 members in vertebrate, has been shown to regulate different developmental processes from Drosophila to humans. This paper suggests that Wnt proteins can act as potential players in the developmental regulation of growth plate assembly and bone development. Indeed, it was recently shown that the strength of $\mathrm{Wnt} / \beta$-catenin signaling determines if mesenchymal cells differentiate into chondrocytes or osteoblasts [19]. Data presented in this paper show that active $\mathrm{Wnt} / \beta$-catenin signaling causes further differentiation of differentiated (hypertrophic) chondrocytes, while it prevents further differentiation in non-differentiated (resting and proliferative) chondrocytes. It could not be excluded that the developmental manner of Wnt/ $\beta$-catenin signaling could be independent of the stage of chondrocyte differentiation, and rather regulated by other factors, such as the strength of the signaling or cell-matrix interaction.

\section{New hormones}

\section{Expression of SHOX in human fetal and childhood growth plate}

Munns CJF, Haase HR, Crowther LM, Hayes MT, Blaschke R, Rappold G, Glass IA, Batch JA Endocrine Research Unit, Royal Children's Hospital Foundation Research Centre, Brisbane, Australia j.batch@mailbox.uq.edu.au J Clin Endocrinol Metab 2004;89:4130-4135

Background: Deletions or mutations in the SHOX gene correlate to abnormalities in the growth plate leading to short stature and skeletal deformity including Leri-Weil syndrome.

Methods: The aim was to document the ontogeny of SHOX protein expression. Expression of SHOX protein by immunohistochemistry was studied from growth plate sections of 7 postmortem fetuses, 4 control subjects undergoing physiodesis and 4 individuals at physiolysis for correction of bilateral Madelung deformity (having SHOX haploinsufficiency).

Results: SHOX protein was found in the reserve, proliferative and hypertrophic zones of human fetal growth plates obtained from 12 weeks of gestation to term and in childhood controls and in Leri-Weil growth plates. No difference in the pattern of SHOX protein or mRNA expression was seen between the control and Leri-Weil growth plates.

Conclusion: The findings suggest that SHOX plays a role in chondrocyte function in the growth plate and that the expression has a developmentally specific pattern. The SHOX protein is expressed in the human growth plate already from 12 weeks of gestation up to the time of growth plate fusion during late adolescence.

SHOX protein is known to us from its role in Turner syndrome. It seems to be involved in the unique radiological feature known as Madelung deformity, affecting the medial side of both radius and ulna. It is a cell type-specific transcriptional activator and its expression in chondrocytes was recently shown to lead to cell cycle arrest and apoptosis [20]. In some individuals with unexplained short stature and SHOX haploinsufficiency, a typical pattern of skeletal disproportions and wrist deformities have been reported [21]. In this paper, SHOX protein was shown to be expressed in fetal and childhood growth plates and the expression was shown to be developmentally regulated. These data suggest that SHOX is an important gene already in early chondrogenesis and skeletal growth. The authors speculate that SHOX may play a role in chondrocyte stacking within the proliferative zone and affect the process of chondrocyte differentiation. The exact role of the SHOX protein in the growth plate needs to be further defined. An improved understanding of the function of the SHOX gene, its regulators, downstream targets, and subsequent interactions could help to explain the multiple phenotypes associated with SHOX haploinsufficiency. It may also provide opportunities for novel therapies to be developed for subjects with Turner syndrome or SHOX mutations and associated short stature and skeletal abnormalities. 


\section{Estrogen regulation of growth hormone action}

Leung K-C, Johannsson G, Leong GM, Ho KKY

Pituitary Research Unit, Garvan Institute of Medical Research, St. Vincent's Hospital, Sydney, Australia

k.ho@garvan.org.au

Endocr Rev 2004;25:693-721

Background: Body growth and metabolism is regulated by growth hormone $(\mathrm{GH})$, a process which is modulated by sex steroids. The interplay between estrogen and GH leads to attainment of gender-specific body composition during puberty. The underline mechanisms of this interaction are not well understood. Much attention has been focused on the effects of estrogen on GH secretion although there is strong evidence that estrogen also modulates $\mathrm{GH}$ action.

Conclusions: Oral but not transdermal administration of estrogen impairs the metabolic action of GH in the liver, causing a fall in IGF-I production and fat oxidation. This leads to a loss of lean tissue and a gain of body fat in postmenopausal women and an impairment of GH effect in hypopituitary women on GH replacement. Estrogen affects GH action also at the level of receptor expression and signaling. Estrogen has been shown to inhibit Janus kinase/STAT-signaling by GH via the induction of SOCS-2, a protein inhibitor for cytokine signaling. This steroid regulation of cytokine receptors is likely to have significance for a diverse range of cytokine function.

In this review, the authors have presented an update on the present perception of estrogen regulation of the effects of $\mathrm{GH}$. We knew that estrogens modulate the $\mathrm{GH}$ receptor (GHR) expression, but over the last years we also learnt that estrogen attenuates $\mathrm{GH}$ action by suppressing GHR post-receptor signaling. The GHR signals through the JAK/STAT pathway, which is negatively regulated by SOCS proteins. The proposed inhibition by estrogen of GHR signaling via up-regulation of SOCS-2 represents a novel paradigm of steroid hormone regulation of cytokine receptor function. Estrogen has already been shown to inhibit prolactin and IL- 6 receptor signaling. There are many physiological observations, which may be explained by estrogen regulation of cytokine action. Thus, the inhibitory regulation of GHR signaling by estrogen mediated through the SOCS proteins is likely to be significant for the regulation of a diverse range of hormone and cytokine functions.

\section{Food for thought}

\section{The endogenous growth hormone secretagogue (Ghrelin) is synthesized and secreted by chondrocytes}

Caminos JE, Gualillo O, Lago F, Otero M, Blanco M, Gallego R, Garcia-Caballero T, Goldring MB, Casanueva FF, Gomez-Reino JJ, Dieguez C

Complexo Hospitalario Universitario de Santiago, Molecular and Cellular Cardiology Unit,

Santiago de Compostela, Spain

gualillo@usc.es

Endocrinology 2005;146:1285-1292

Background: Ghrelin is the recently isolated endogenous ligand for the GH secretagogue receptor (GHS$\mathrm{R})$. It is mainly expressed in the stomach but it has also been demonstrated in other tissues such as hypothalamus and placenta. Ghrelin acts at a central level to stimulate $\mathrm{GH}$ secretion and to regulate food intake. However, any role of Ghrelin in the local regulation of growth plate cartilage is unknown. Methods: Specimens of rat epiphyseal growth plates, mouse and human tissues, as well as the ATDC-5 murine chondrogenic cell line and immortalized human juvenile costal chondrocyte cell lines (T/C28a2, C-20/A, C28/12) were used. RT-PCR and immunohistochemistry were used for mRNA and protein expression determinations, respectively.

Results: Ghrelin peptide and mRNA were found to be expressed in human, mouse, and rat chondrocytes. Immunoreactive Ghrelin was localized mainly in the proliferative and hypertrophic zones of the growth 
plate, and in mouse and human chondrocytic cell lines. Expression of Ghrelin mRNA was shown in rat cartilage and in mouse and human chondrocytes cell lines. Ghrelin mRNA expression was detected in fetal and postnatal (days 1, 15 and 25 of age) rats. The functional GH secretagogue receptor type 1A was not detectable by RT-PCR. However, binding analysis with ${ }^{125} \mathrm{I}$ Ghrelin suggested the presence of unidentified receptors. Scatchard analysis suggested the presence of two receptors; one with high and one with low affinity. Finally, Ghrelin was able to stimulate cAMP production, to inhibit chondrocyte metabolic activity and to decrease both spontaneous and insulin-induced uptakes of long chain fatty acids in both human and murine chondrocyte cell lines.

Conclusion: This study for the first time demonstrates the expression of Ghrelin by chondrocytes and suggests novel potential roles for this hormone in cartilage homeostasis.

Ghrelin is the endogenous ligand for growth hormone (GH) secretagogue receptor (GHS-R). Interestingly, it was recently shown that Ghrelin may directly stimulate bone formation [22]. This paper for the first time demonstrates the expression of Ghrelin in growth plate cartilage. The expression was localized in both proliferative and maturing chondrocytes, which suggests a potential role for Ghrelin in the regulation of longitudinal bone growth. Although GHS-R 1A was not expressed in the growth plate, binding of radiolabeled Ghrelin was observed. This together with Ghrelin-dependent stimulation of CAMP production and inhibition of chondrocyte metabolic activity, suggest that yet unidentified Ghrelin receptors may be expressed in growth plate chondrocytes. There is a wellknown connection between food intake and bone elongation and it could be speculated that Ghrelin is a potential candidate for such a connection. The present data suggest additional effects of Ghrelin mediated through direct actions in the growth plate cartilage. However, it should be pointed out that the growth plate is a complicated organ with several autocrine/paracrine factors involved in a complex cross-talk between the different zones. The approach in this paper, using immortalized cell lines, is a simplified one. The data have to be confirmed in experimental models where the spatial organization within the growth plate is intact, e.g. using growth plate/metatarsal organ cultures or in vivo experiments giving unilateral injections of Ghrelin. Thus, additional studies are needed before the exact role of Ghrelin in the regulation of growth plate cartilage can be defined.

References

1. Serizawa N: Initial characterization of a new miniature animal model in the rat: studies on anatomy, pituitary hormones and GH mRNA in miniature rat Ishikawa (in Japanese). Nippon Naibunpi Gakkai Zasshi 1993;69:33-45.

2. Pfeifer A, Aszodi A, Seidler U, Ruth P, Hofmann F, Fassler R: Intestinal secretory defects and dwarfism in mice lacking cGMP-dependent protein kinase II. Science 1996;274:2082-2086.

3. Hayashi YK, Chou FL, Engvall E, Ogawa M, Matsuda C, Hirabayashi S, Yokochi K, Ziober BL, Kramer RH, Kaufman SJ, Ozawa E, Goto Y, Nonaka I, Tsukahara T, Wang JZ, Hoffman EP, Arahata K: Mutations in the integrin $\alpha 7$ gene cause congenital myopathy. Nat Genet 1998;19:94-97.

4. Kennedy AM, Christie PT, Harding B, Pannett AAJ, Dearlove A, Whyte MP, Thakker RV: Matrix metalloproteinase 13 (MMP13) causes spondyloepimetaphyseal dysplasia (SEMD), Missouri variant. Endocr Abstr 2003;5:OC40.

5. Schmeling H, Seliger E, Horneff G: Growth reconstitution in juvenile idiopathic arthritis treated with etanercept. Clin Exp Rheumatol 2003;21:779-784.

6. Cezard J, Nouaili N, Talbotec C, Hugot J, Gobert J, Schmitz J, Mougenot J, Alberti C, Goulet O: A prospective study of the efficacy and tolerance of a chimeric antibody to tumor necrosis factors (remicade) in severe pediatric Crohn disease. J Pediatr Gastroenterol Nutr 2003;36:632-636.

7. Goldzieher MA: Treatment of excessive growth in the adolescent female. J Clin Endocrinol Metab 1956;16:249-252.

8. Nilsson O, Chrysis D, Pajulo O, Boman A, Holst M, Rubinstein J, Martin Ritzen E, Savendahl L: Localization of estrogen receptors- $\alpha$ and $-\beta$ and androgen receptor in the human growth plate at different pubertal stages. J Endocrinol 2003;177:319-326.

9. Smith EP, Boyd J, Frank GR, Takahashi H, Cohen RM, Specker B, Williams TC, Lubahn DB, Korach KS: Estrogen resistance caused by a mutation in the estrogen-receptor gene in a man. N Engl J Med 1994;331:1056-1061.

10. Drop SLS, de Waal WJ, de Muinck Keizer-Schrama SMPF: Sex steroid treatment of constitutionally tall stature. Endocr Rev 1998;19:540-558.

11. Woods K, Camacho-Hubne RC, Barter D, Clark A, Savage M: Insulin-like growth factor I gene deletion causing intrauterine growth retardation and severe short stature. Acta Paediatr Suppl 1997;423:39-41.

12. Pescovitz OH, Comite F, Cassorla F, Dwyer AJ, Poth MA, Sperling MA, Hench K, McNemar A, Skerda M, Loriaux DL, et al: True precocious puberty complicating congenital adrenal hyperplasia: treatment with a luteinizing hormone-releasing hormone analog. J Clin Endocrinol Metab 1984;58:857-861.

13. Soliman AT, AlLamki M, AlSalmi I, Asfour M: Congenital adrenal hyperplasia complicated by central precocious puberty: linear growth during infancy and treatment with gonadotropin-releasing hormone analog. 1997;46:513-517.

14. Quintos JBQ, Vogiatzi MG, Harbison MD, New MI: Growth hormone therapy alone or in combination with gonadotropin-releasing hormone analog therapy to improve the height deficit in children with congenital adrenal hyperplasia. J Clin Endocrinol Metab 2001;86:1511-1517.

15. Kronenberg HM: Developmental regulation of the growth plate. Nature 2003;423:332.

16. Karsenty G, Wagner EF: Reaching a genetic and molecular understanding of skeletal development. Dev Cell 2002;2:389. 
17. Zhang CL, McKinsey TA, Chang S, Antos CL, Hill JA, Olson EN: Class II histone deacetylases act as signal-responsive repressors of cardiac hypertrophy. Cell 2002;110:479-488.

18. Chang S, McKinsey TA, Zhang CL, Richardson JA, Hill JA, Olson EN: Histone deacetylases 5 and 9 govern responsiveness of the heart to a subset of stress signals and play redundant roles in heart development. Mol Cell Biol 2004;24:8467-8476.

19. Day TF, Guo X, Garrett-Beal L, Yang Y: Wnt/ß-catenin signaling in mesenchymal progenitors controls osteoblast and chondrocyte differentiation during vertebrate skeletogenesis. Dev Cell 2005;8:739-750.

20. Marchini A, Marttila T, Winter A, Caldeira S, Malanchi I, Blaschke RJ, Hacker B, Rao E, Karperien M, Wit JM, Richter W, Tommasino M, Rappold GA: The short stature homeodomain protein SHOX induces cellular growth arrest and apoptosis and is expressed in human growth plate chondrocytes. J Biol Chem 2004;279:37103-37114.

21. Binder G, Ranke MB, Martin DD: Auxology is a valuable instrument for the clinical diagnosis of SHOX haploinsufficiency in school-age children with unexplained short stature. J Clin Endocrinol Metab 2003;88:4891-4896.

22. Fukushima N, Hanada R, Teranishi H, Fukue Y, Tachibana T, Ishikawa H, Takeda S, Takeuchi Y, Fukumoto S, Kangawa K, Nagata K, Kojima M: Ghrelin directly regulates bone formation. J Bone Miner Res 2005;20:790-798. 